\title{
The effect of soil stiffness variations on Tunnel Lining Internal Forces under seismic loading and Case comparison with existing analytical methods
}

\author{
Reza Zamani', M.R. Motahari ${ }^{2, *}$ \\ ${ }^{1}$ Department of civil engineering, Khomein Branch, Islamic Azad University, Khomein, Iran \\ ${ }^{2}$ Dept. of Civil Engineering, Faculty of Engineering, Arak University, Arak, Iran \\ *m-motahari@araku.ac.ir
}

\begin{abstract}
Closed-form solutions are of great interest for quick and accurate evaluation of stresses and displacements around underground excavations. Dynamic effects on underground structures have often been neglected based on the assumption that their response to earthquake loading is relatively safe as compared to that of surface structures. Nevertheless, several examples of recorded damage to underground structures for which seismic forces were not considered in the original design. Therefore, during the seismic excitement, tunnels are basically subjected to three kinds of deformation : axial compression and extension, longitudinal bending and ovaling or racking. In the present investigation a series of numerical analyses under the influence of soil hardness parameters based on no-slip between soil and tunnel lining were performed, using finite element code PLAXIS, and outputs were compared with result of analytical solutions.
\end{abstract}

Keywords: Closed-form solution, finite element method, Tunnel Lining, maximum shear strain 


\section{Introduction:}

Underground openings in soils and rocks are excavated for a variety of purposes and in a wide range of sizes, ranging from boreholes through tunnels, drifts, cross-cuts and shafts to large excavations such as caverns, etc. A feature common to all these openings is that the release of pre-existing stress upon excavation of the opening will cause the soil or rock to deform elastically at the very least. However, if the stresses around the opening are not high enough then the rock will not deform in an inelastic manner. This is possible for shallow openings in relative competent geomaterials where high tectonic stresses are absent. An understanding of the manner in which the soil or rock around a tunnel deforms elastically due to changes in stress is quite important for underground engineering problems. In fact, the accurate prediction of the in situ stress field and deformability moduli through back-analysis of tunnel convergence measurements and of the 'Ground Reaction Curve' is essential to the proper design of support elements for tunnels [Hoek and Brown,1980; Brady and Brown,1985].

The availability of many accurate and easy to use finite element, finite difference, or boundary element computer codes makes easy the stressdeformation analysis of underground excavations. However, Carranza- Torres and Fairhurst note explicitly in their paper [Carranza-Tores and Fairhurst, 1999].

One of the simplifying assumptions always made by various investigators during studying-usually in a preliminary design stage-analytically stresses and displacements around a tunnel is that it has a circular crosssection [Hoek and Brown,1980; Brady and Brown,1985; Sulem et al, 1987]. This is due to the fact that the celebrated Kirsch's [Kirsch, 1898] analytical solution of the circular cylindrical opening in linear elastic medium is available in the literature and it is rather simple for calculations [Jaeger, 1976]. On the other hand, Gercek in 1991 and 1997 was the first investigator who presented a closed-form solution for the stresses around tunnels with arched roofs and with either flat or parabolic floor having an axis of symmetry and excavated in elastic media subjected to an arbitrarily oriented in situ far-field biaxial stress state.
Gercek has used the method of conformal mapping and Kolosov-Muskhelishvili in 1963 complex potentials along with the "modified method of undetermined coefficients" of Chernykh [Novozhilov, 1961]. However, Gercek did not consider:

(a) The incremental release of stresses due to excavation of the tunnel,

(b) The solution for the displacements,

(c) The influence of support pressure on tunnel walls on the stresses and displacements, and

(d) The methodology to derive the constant coefficients of the series representation of the conformal mapping for prescribed tunnel crosssections.

DING De-yun et al in 2010, investigated on Prediction of vibrations from underground trains on Beijing metro line 15, and the numerical results show that the influence of vibrations from underground trains on sensitive equipment depends on the track types. At frequencies above $10 \mathrm{~Hz}$, the floating slab track with a natural frequency of $7 \mathrm{~Hz}$ can be effective to attenuate the vibrations.

Taiyue et al in 2011, conducted research with numerical simulation on strata consolidation subsidence by dewatering, dynamic dewatering, and non-dewatering construction method, taking the integrated effects of fluid-solid coupling and tunneling mechanics into account. We obtained the curved surfaces of ground surface subsidence and strata consolidation subsidence. The results show that the non-dewatering construction method is the most effective method to control the strata consolidation subsidence induced by metro tunneling in saturated soft clay strata, and it has been successfully applied to the construction of the Shenzhen metro line 1.

Zhang et al in 2012 studied on a new closedform solution for circular openings modeled by the Unified Strength Theory and radiusdependent Young's modulus. The new closedform solution obtained in their paper is a series of results rather than one specific solution; hence, it is suitable for a wide range of rock masses and engineering structures. The traditional solutions, which are based on the Mohr-Coulomb failure criterion and the Generalized Twin Shear Stress yield criterion, can be categorized as special cases of this 
proposed solution. The new solution agrees reasonably well with the results of a borehole collapse test, a secondary development numerical simulation and an additional closedform solution using the generalized non-linear Hoek-Brown failure criterion. Parametric studies were conducted to investigate the effects of intermediate principal stress, RDM and dilatancy on the results. It is shown herein that the effects of intermediate principal stress and dilatancy are significant; the RDM model is recommended as the optimum approach for calculating radial displacement and support pressure.

Also Manh Vu et al in 2013 studied about Semi-Analytical Solution for Stresses and Displacements in a Tunnel Excavated in Transversely Isotropic Formation with NonLinear Behavior.

Dong $\mathrm{Wu}$ et al. in 2013 were investigate on dynamic analysis of tunnel entrance under the effect of Rayleigh wave and they result that The analysis result matches well with part of field observation for damaged tunnels entrance in Wenchuan Earthquake, which indicates that the effect of Rayleigh wave should be paid sufficient attention in the research on seismic performance of tunnel entrance.

S.A. Mazek et al in 2013 used A 2-D finite element analysis to understand the performance of the Cairo metro tunnel system-Line 3. The analysis takes into account the change in stress, the non-linear behavior of the soil, and the construction progress .

Also Angelo Amorosi et al in 2014, investigated on Numerical prediction of tunnel performance during centrifuge dynamic tests, and a comparison between numerical analyses and centrifuge test results relative to the seismic performance of a circular tunnel is provided. The comparison between numerical simulations and measurements is presented in terms of acceleration histories and Fourier spectra as well as of profiles of maximum acceleration along free-field and near-tunnel verticals. In addition, loading histories of normal stress and bending moments acting in the tunnel lining were considered.

\section{RESPONSE OF TUNNEL TO THE SPREAD WAVES FROM EARTHQUAKE AND} SCRUTINY OF CHANGE IN PRODUCT
Response of tunnel to the ground vibrations caused by the movement of the waves can be classified into three main categories below:

a) Axial and Curvature Deformations

b) Oval shape deformation (circular cross section)

c) Rotation deformation or rocking (Rectangular cross section)

In horseshoe tunnel, distortion of crosssection as a combination of circular and rectangular sections deformations occur. In the following explanation is provided about each of these deformations.

\section{A. Axial and Curvature Deformations}

Axial and curvature deformations develop in a horizontal or nearly horizontal linear tunnel (such as most tunnels) when seismic waves propagate either parallel or obliquely to the tunnel. The tunnel lining design considerations for these types of deformations are basically in the longitudinal direction along the tunnel axis. Figure 1 shows the idealized representations of axial and curvature deformations. The general behavior of the linear tunnel is similar to that of an elastic beam subject to deformations or strains imposed by the surrounding ground [Wang; 1993].
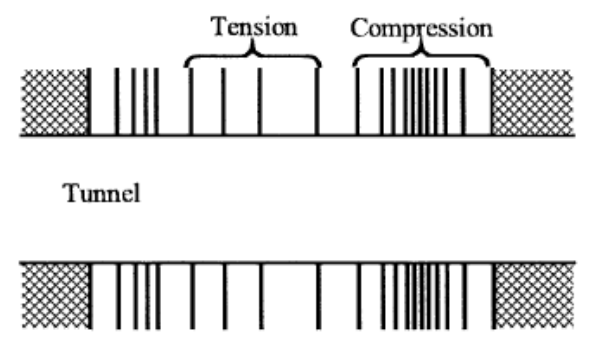

(a)

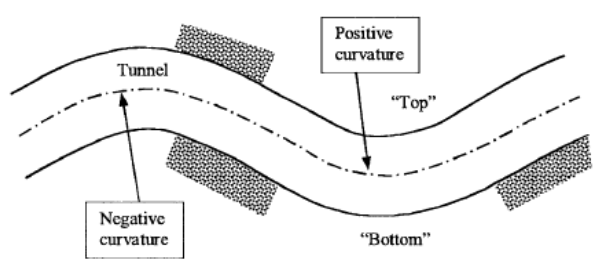

(b)

Figure1- idealized representations of (a) Axial Deformation, (b) Curvature Deformation

To determine the axial and Curvature deformations can be used one-dimensional models. Perhaps the simplest way to consider this tunnel as a structural beam. But for larger 
tunnels, it is necessary that three dimensional models used to estimate these deformations.

\section{B. Ovaling or Racking Deformations}

The ovaling or racking deformations of a tunnel structure may develop when waves propagate in a direction perpendicular or nearly perpendicular to the tunnel axis, resulting in a distortion of the cross-sectional shape of the tunnel lining. Design considerations for this type of deformation are in the transverse direction .

Figure 2 shows the ovaling distortion and racking deformation associated with circular tunnels and rectangular tunnels, respectively. The general behavior of the lining may be simulated as a buried structure subject to ground deformations under a two-dimensional, planestrain condition.

ovaling and racking deformations may be caused by vertically, horizontally or obliquely propagating seismic waves of any type. Many previous studies have suggested, however, that the vertically propagating shear wave is the predominant form of earthquake loading that governs the tunnel lining design against ovaling/racking. The following reasons are given:

Ground motion in the vertical direction is generally considered less severe than its horizontal component. Typically, vertical ground motion parameters are assumed to be $1 / 2$ to $2 / 3$ of the horizontal ones. (Note that a vertically propagating shear wave causes the ground to shake in the horizontal direction.) This relation is based on observation of California earthquakes, which are most commonly of the strike-slip variety in which horizontal motion predominates

For thrust faults, in which one rock block overrides another, vertical effects may equal or exceed the horizontal ones. The effects of thrust faulting are usually more localized, however, than those of the strike-slip faulting, and they are attenuated more rapidly with distance from the focus .

For tunnels embedded in soils or weak media, the horizontal motion associated with vertically propagating shear waves tends to be amplified. In contrast, the ground strains due to horizontally propagating waves are found to be strongly influenced by the ground strains in the rock beneath. Generally, the resulting strains are smaller than those calculated using the properties of the soils .

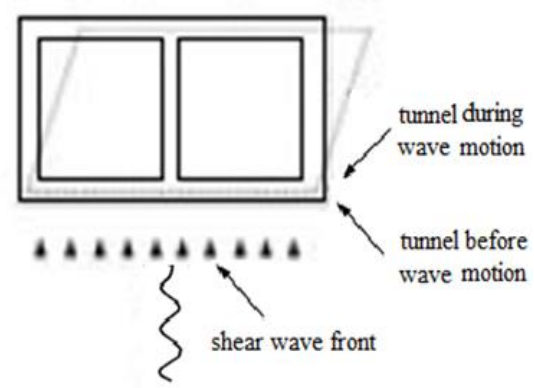

(a)

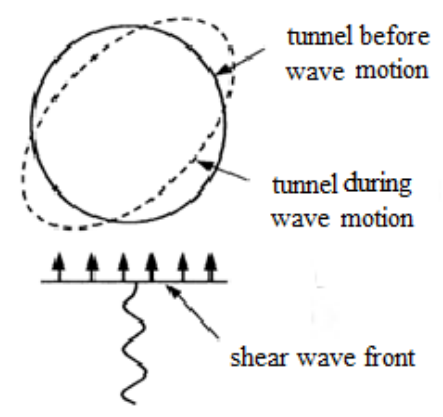

(b)

Figure2- (a) Racking Deformation of a Rectangular Cross Section (b) Ovaling Deformation of a Circular Cross Section

\section{Analytical solutions of ovaling deformation of circular tunnel with soil-structure interaction}

The simplest form of estimating ovaling deformation is to assume the deformations in a circular tunnel to be identical to "free-field", thereby ignoring the tunnel- ground interaction. This assumption is appropriate when the ovaling stiffness of the lined tunnel is equal to that of the surrounding ground .

The circular tunnel-ground shearing is then modeled as a continuous medium (referred to as non-perforated ground) without the presence of the tunnel (Fig. 3), in which the diametric strain for a circular section is calculated as Eq (1) in Hashash et al. (2001) : 


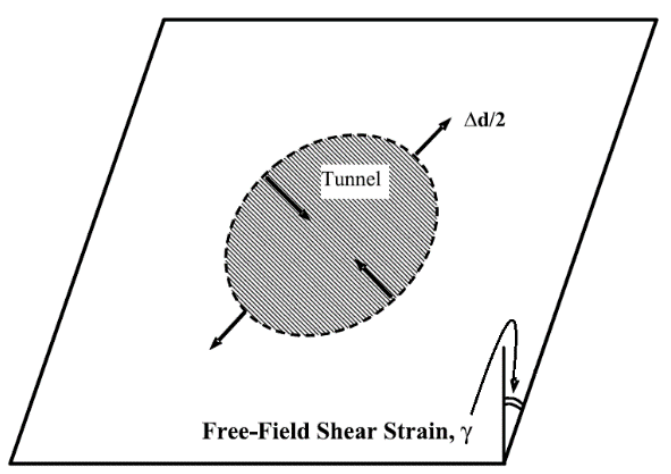

Figure3- Free-field shear distortion of perforated (tunnel cavity is empty) and nonperforated ground (tunnel cavity is filled), circular shape

$\frac{\Delta \mathrm{d}_{\text {free-field }}}{\mathrm{d}}= \pm \frac{\gamma_{\max }}{2}$

If the ovalling stiffness is very small compared to the surrounding ground, the tunnel distortion or diametric strain is calculated assuming an unlined tunnel as Eq (2) in Hashash et al. (2001), (referred to as perforated ground):

$\frac{\Delta \mathrm{d}_{\text {free-field }}}{\mathrm{d}}= \pm 2 \gamma_{\max }\left(1-v_{m}\right)$

That $\Delta \mathrm{d}_{\text {free-field }}$ is Free-field diametric deflection in non-perforated ground, $\gamma_{\max }$ is maximum free-field shear strain of soil or rock medium and $v_{m}$ is Poisson's ratio of soil or rock medium and this deformation is much greater in the case where the presence of the tunnel is included compared to the case where only the continuous ground deformation is assumed.

Also, the maximum shear strain in the medium for both constant shear strain and variable shear strain may be obtained as Hashash et al. (2005):

$$
\begin{aligned}
& G_{m}=\frac{E_{m}}{2\left(1+v_{m}\right)} \\
& C_{m}=\sqrt{\frac{G_{m}}{\rho_{m}}} \\
& \gamma_{\max }=\frac{V s}{C_{m}}
\end{aligned}
$$

Where $\mathrm{Q} m=\boldsymbol{\gamma}_{s} / \mathrm{g}, \mathrm{Gm}$ is shear modulus, $V_{S}$ is peak particle velocity associated with $S$ waves and $\mathrm{C}_{\mathrm{m}}$ is Apparent velocity of S-wave propagation in soil. Full-slip assumption under simple shear deformation, however, may cause significant underestimation of the maximum thrust. Therefore, based on the above equations, the modeling is carried out as follows.

\section{Numerical modeling:}

The finite element program Plaxis v8 was used to develop a numerical model of a reference problem to study effects of soil stiffness on Tunnel Lining. The analyses use assumptions identical to those of the analytical solution; (a) plane-strain condition, (b) ground and lining are linear elastic and mass-less materials. Shear loading is applied at the upper ends of the boundaries to simulate pure shear condition. Also, based on the closed form solution and also considering the geometry $40 \times 100$ meters and physical properties of the original tunnel is including $\mathrm{d}=6 \mathrm{~m}$ at a depth of 15 meters above ground level that shown in figure $4, \gamma_{\max } 0(2) 252$ and Properties of different materials are shown in Table 1.

Table 1- parameters of Soil

\begin{tabular}{|c|c|c|}
\hline $\begin{array}{c}\text { modulus of } \\
\text { elasticity }\end{array}$ & Poisson's ratio & materials \\
\cline { 1 - 2 } $\mathrm{E}\left(\mathrm{KN} / \mathrm{m}^{2}\right)$ & 0 & \multirow{2}{*}{ linear elastic } \\
\hline 312000 & 0.3 & \\
\hline
\end{tabular}

Table 2- parameters of tunnel

\begin{tabular}{|c|c|c|}
\hline Unit & values & $\begin{array}{c}\text { lining } \\
\text { parameters }\end{array}$ \\
\hline$\left(\mathrm{KN} / \mathrm{m}^{2}\right)$ & 24800000 & $\begin{array}{c}\text { modulus of } \\
\text { elasticity }\end{array}$ \\
\hline $\mathrm{m}^{2} / \mathrm{m}$ & 0.3 & Area \\
\hline $\mathrm{m}^{4} / \mathrm{m}$ & 0.00225 & $\begin{array}{c}\text { moment of } \\
\text { inertia }\end{array}$ \\
\hline $\mathrm{m}$ & 0.3 & thickness \\
\hline----- & 0.2 & Poisson's ratio \\
\hline
\end{tabular}




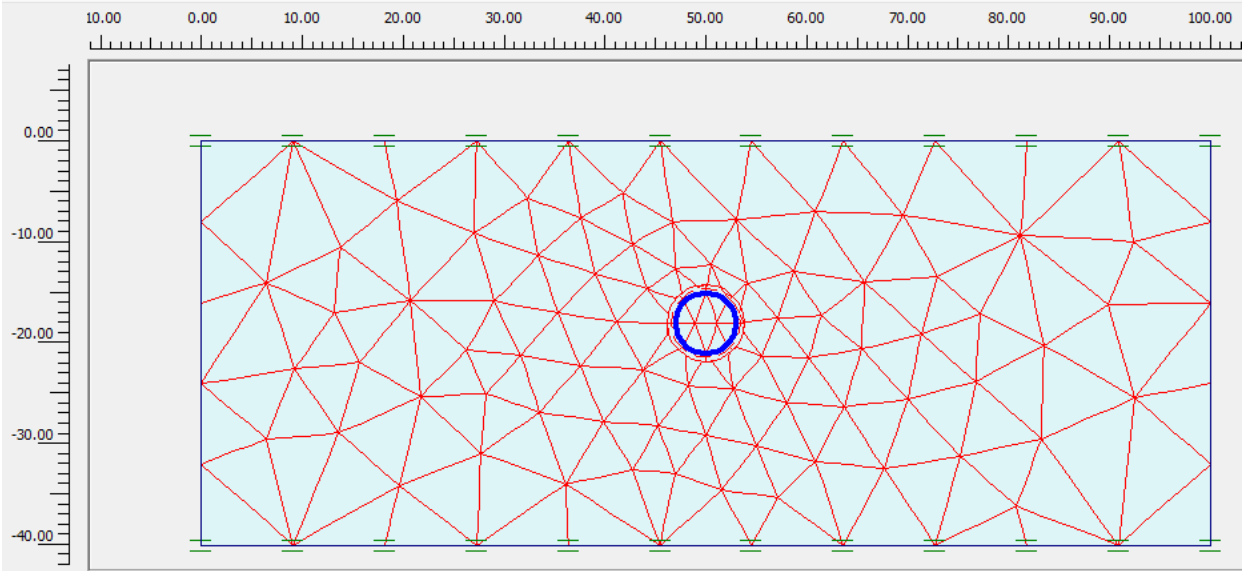

Figure 4. Location of the tunnel with mesh for validation

With geometry of the model And after assign the materials, then applying initial conditions Turn to step of calculation. Then by applying the shear strain, deformation of output is as follows. The deformed mesh can be seen in Figure 5.

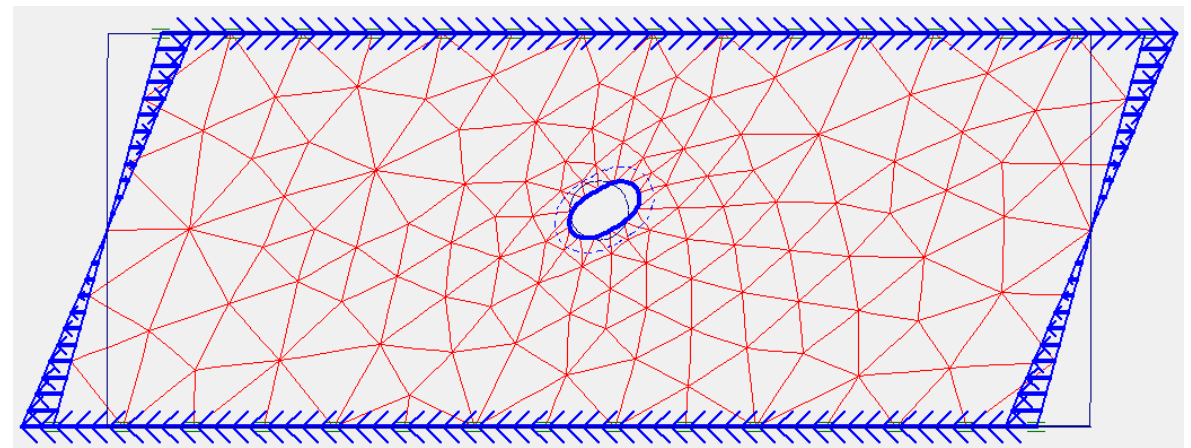

Figure 5. deformed mesh geometry of the model

After showing changes in the soil, caused by the application of shear strain, changes in the tunnel lining is shown in Figure 6.

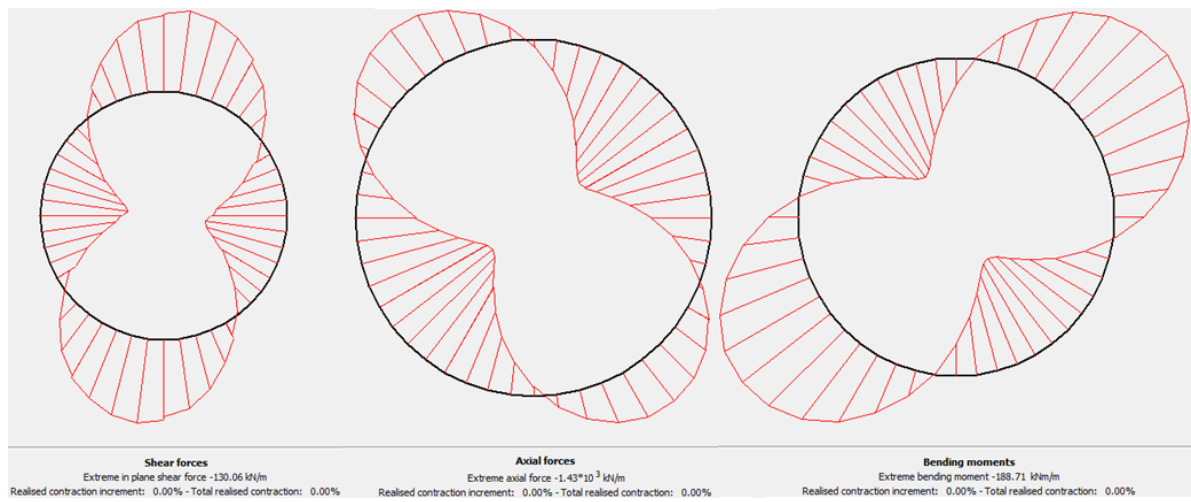

Figure 6: axial force, shear force and bending moment created in the tunnel lining

After showing changes in the tunnel lining, the effect of shear strain on shear force, bending moments and axial force are shown in Figures 7 to 9 . 


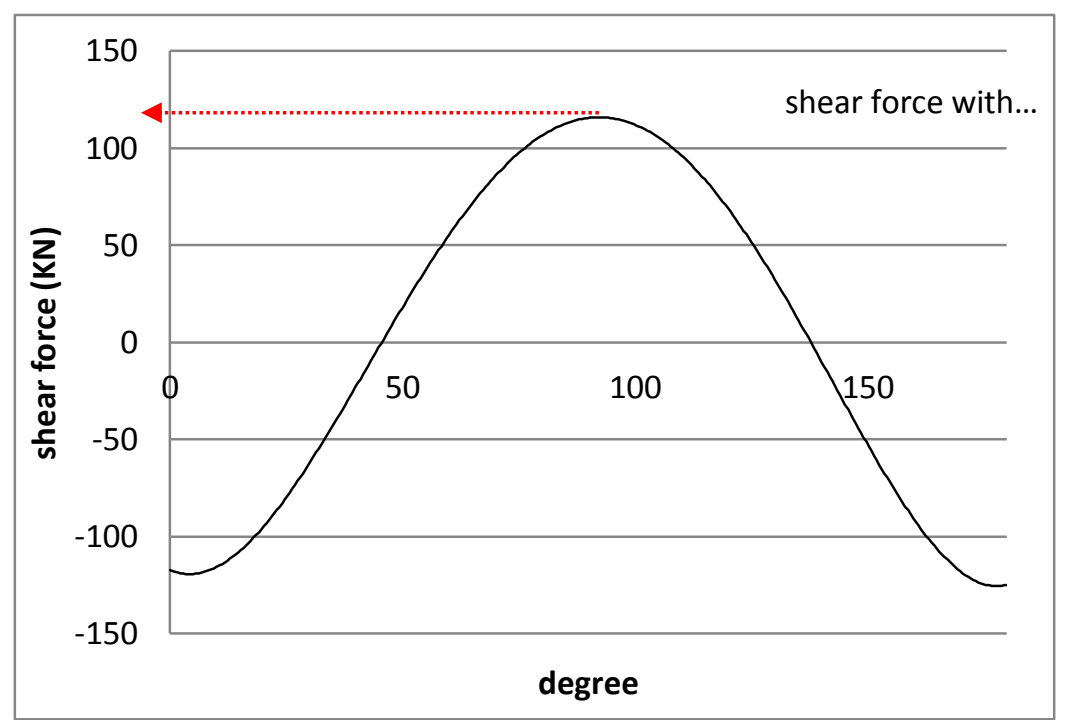

Figure 7. numerical analysis of shear force for interaction between the tunnel and ground

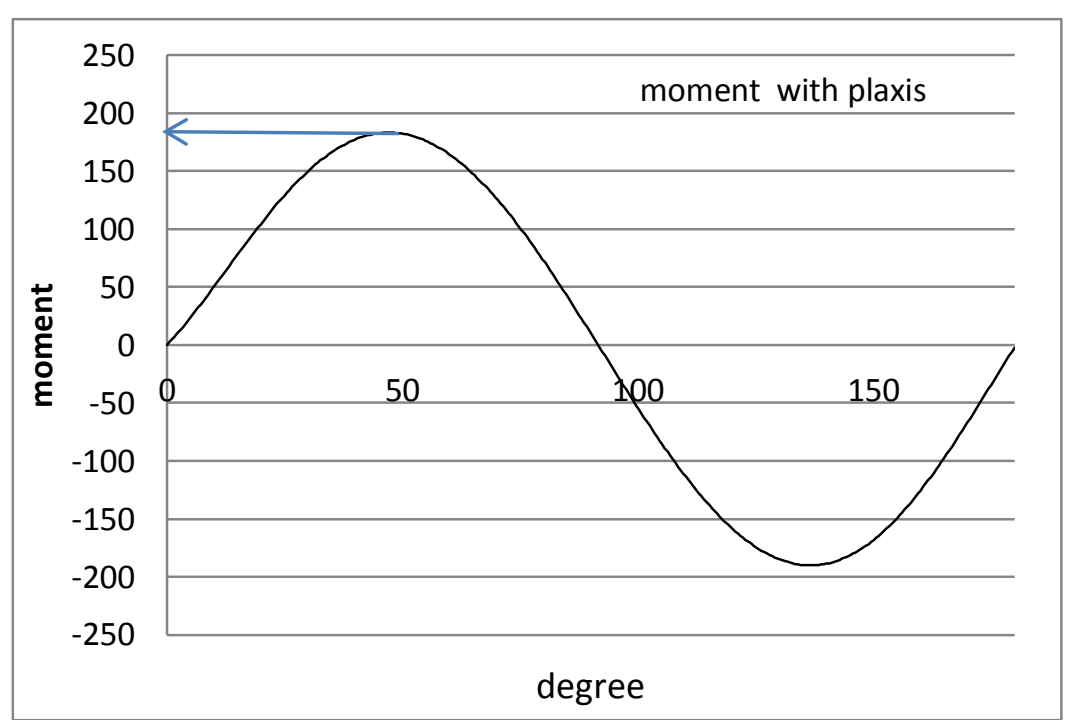

Figure 8. numerical analysis of bending moment for interaction between the tunnel and ground

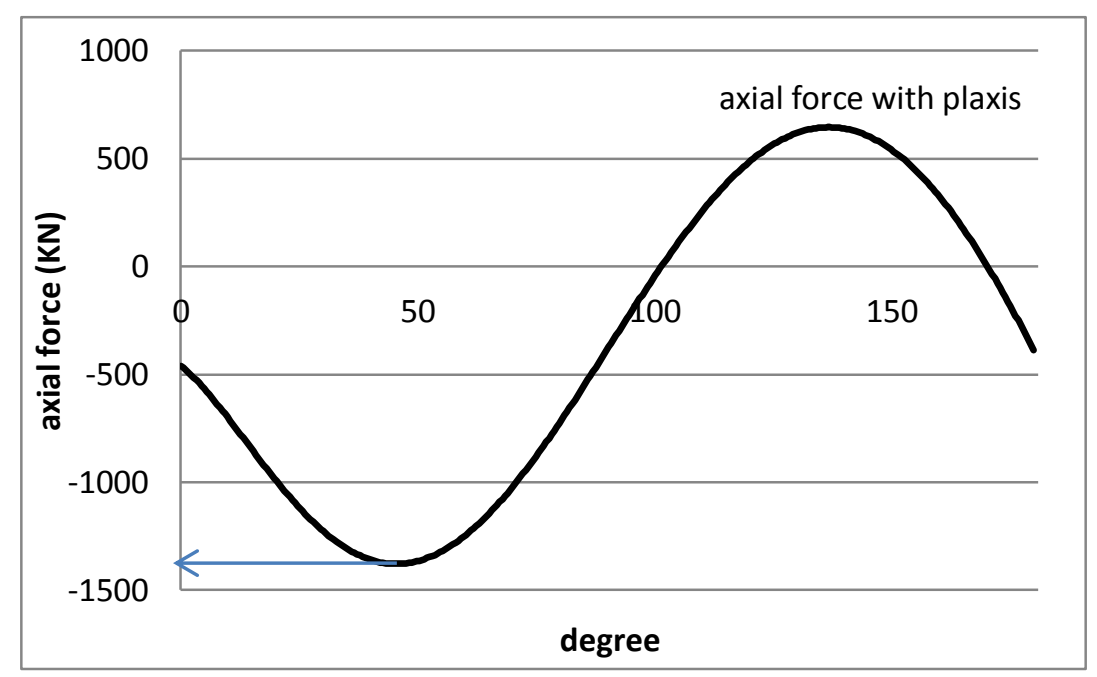

Figure 9. numerical analysis of axial force for interaction between the tunnel and ground 
As shown by the arrows, maximum axial force, bending moment and shear force to compare with the results of wang, bobet and Penzien shown in the table 3.

Table 3. Comparison of the numerical results with results of Wang, Bobet and Penzien

\begin{tabular}{|c|c|c|c|c|c|}
\hline Numerical (Plaxis) & bobet & wang & penzien & unit & \\
\hline 1430 & 1046.49 & 1045.37 & 124.63 & $(\mathrm{KN})$ & $\mathrm{T}_{\max }$ \\
\hline 130.06 & ----- & ----- & 124.63 & $(\mathrm{KN})$ & $\mathrm{V}_{\max }$ \\
\hline 188.7 & 159.27 & 188.8 & 186.95 & $(\mathrm{KN}-\mathrm{m})$ & $\mathrm{M}_{\max }$ \\
\hline
\end{tabular}

Thus, as is apparent from Table 3, using the finite element software plaxis for modeling and studying the interaction between soil and tunnel, this software is reliable. It continues paper, parametric studies by changing the soil stiffness properties is done.

Parametric Study change the modulus of elasticity in soil
In this section, by changing the modulus of elasticity of soil to compare the results of tunnel stability will be discussed. Modulus of elasticity in the range of 200000, 312000 and 400000 changed and comparing results is presented in the following graph.

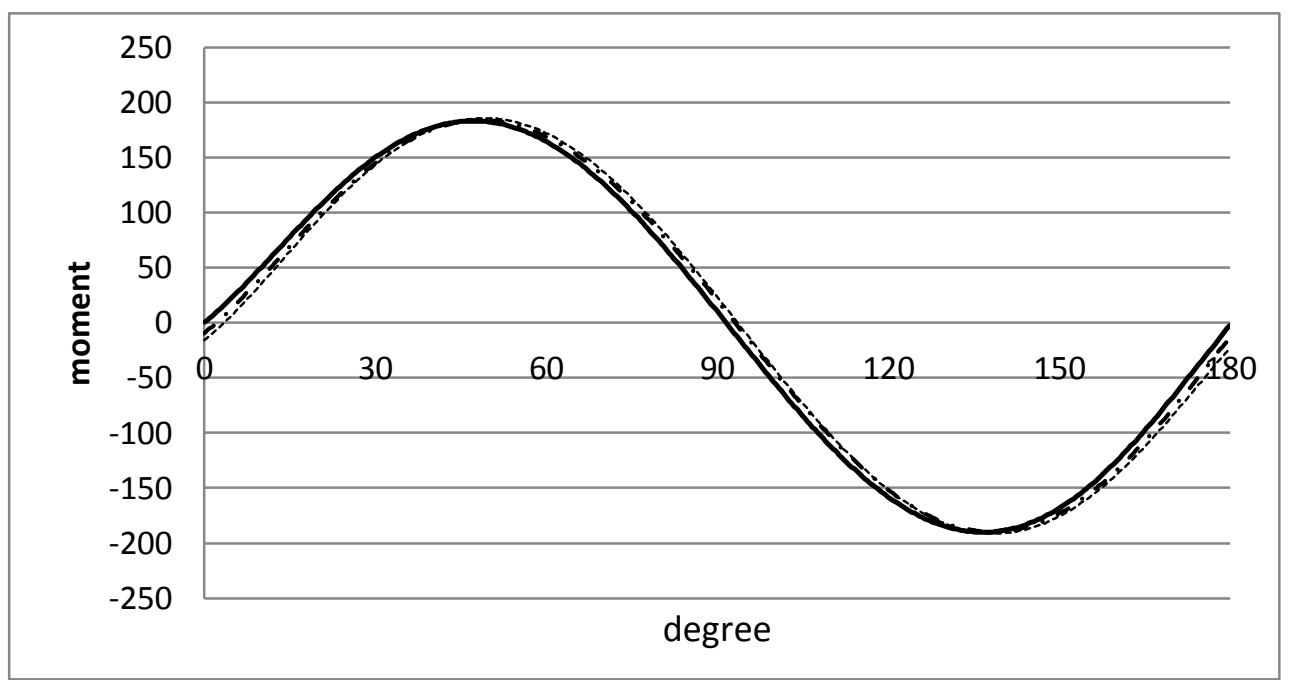

Figure 10-compares the numerical analysis of bending moment, for tunnel and ground interaction, against changing the soil modulus

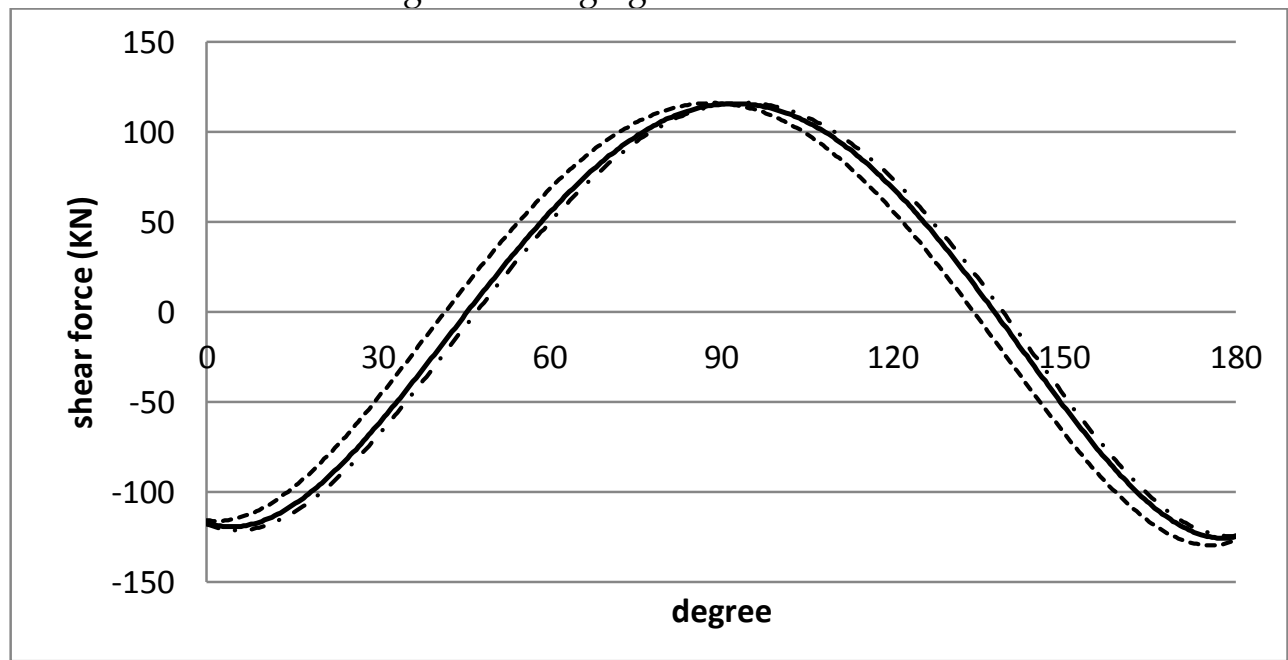

Figure 11- compares the numerical analysis of shear force, for tunnel and ground interaction, against changing the soil modulus 


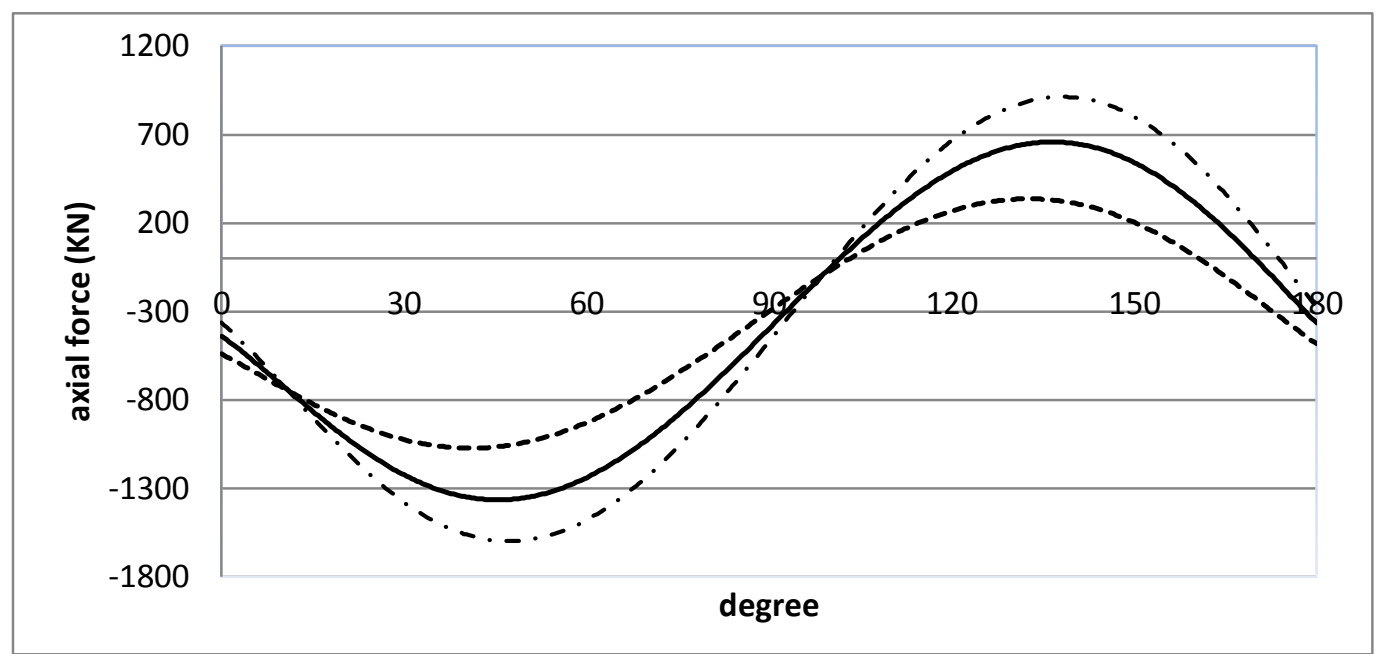

Figure 12- compares the numerical analysis of axial force, for tunnel and ground interaction, against changing the soil modulus

As seen from Figures 10 to 12, with increasing modulus of elasticity, axial force on tunnel lining increased and shear force and bending moment would be associated with subtle changes.

\section{Change in soil Poisson's ratio}

In this section, changing the soil Poisson's ratio comparing results tunnel stability will be discussed. Soil Poisson's ratio in the range of 0.1 , $0.2,0.3$ and 0.4 changed and comparing results is presented in the following graph.

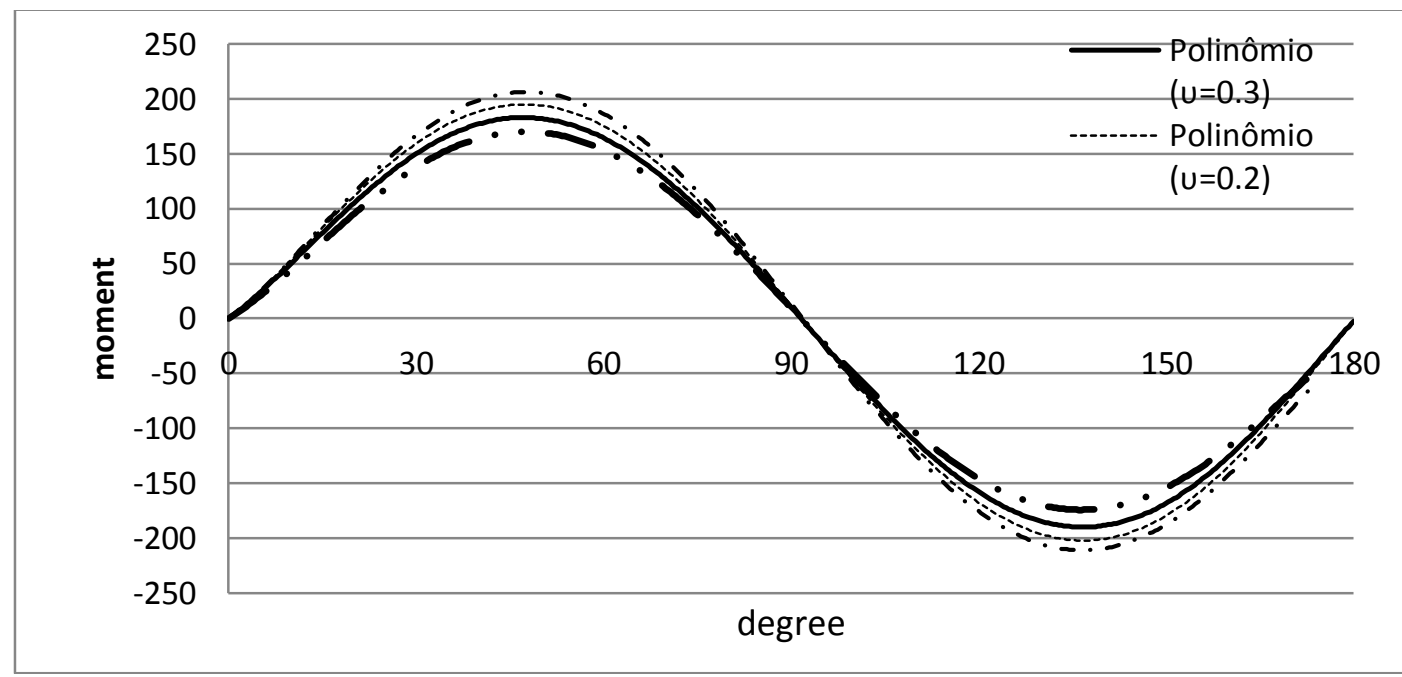

Figure 13- compares the numerical analysis of bending moment, for tunnel and ground interaction, against changing the soil Poisson's ratio 


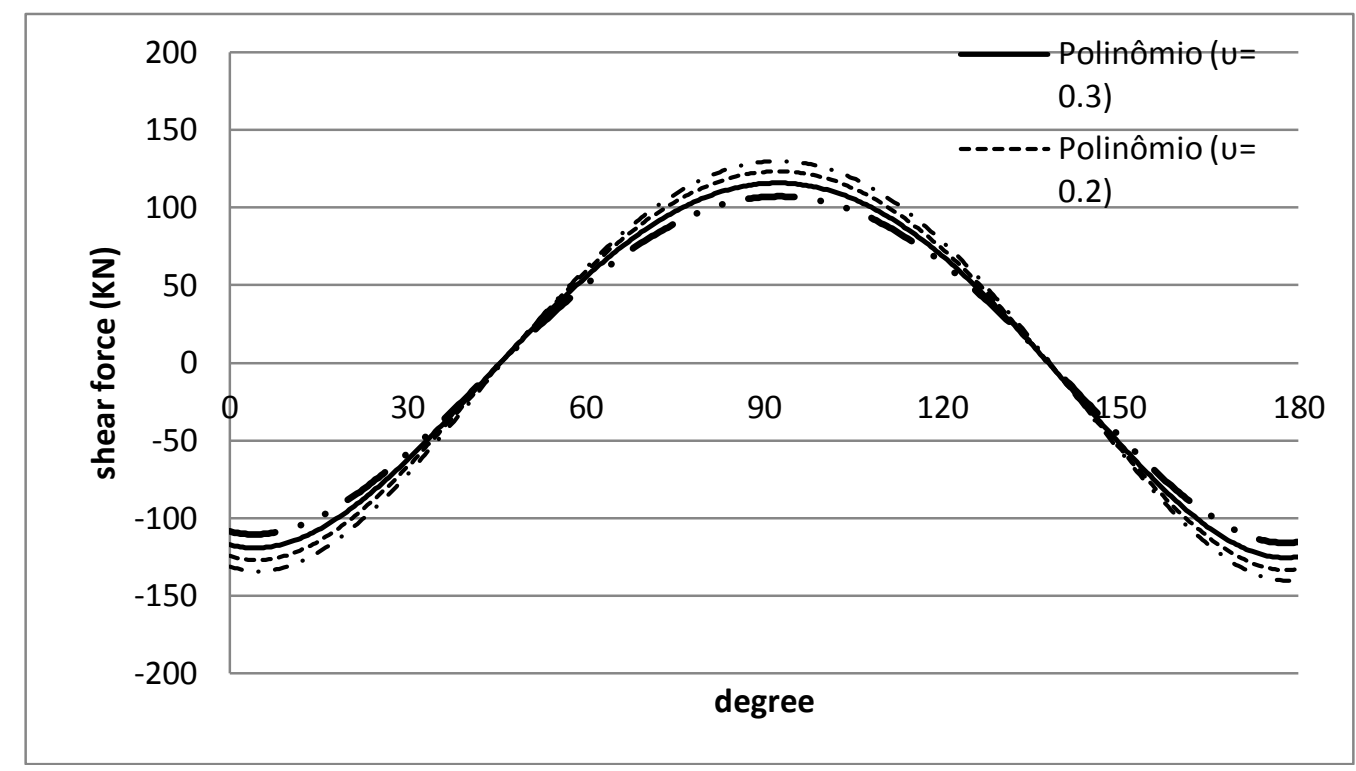

Figure 14- compares the numerical analysis of shear force, for tunnel and ground interaction, against changing the soil Poisson's ratio

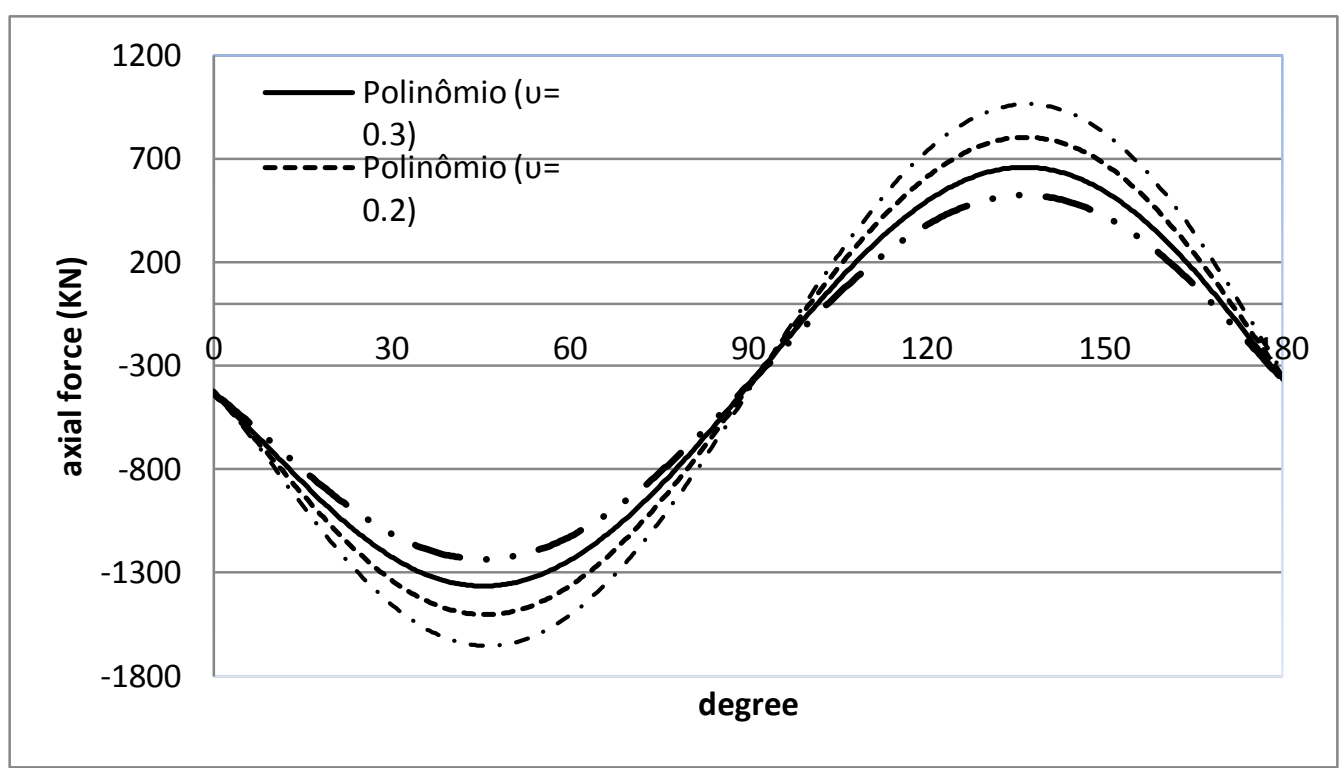

Figure 15- compares the numerical analysis of axial force, for tunnel and ground interaction, against changing the soil Poisson's ratio

As seen from Figures 13 to 15, with increasing soil Poisson's ratio, axial force, shear force and bending moment in tunnel lining decreased.

\section{CONCLUSIONS}

Based on this research considering the original tunnel the following points can be concluded:

1) With increasing modulus of elasticity, axial force on tunnel lining increased and shear force and bending moment would be associated with subtle changes.

2) With increasing soil Poisson's ratio, axial force, shear force and bending moment in tunnel lining decreased.

3) With comparisons closed form solution with numerical method, there is no significant difference between numerical and Wang method in bending moment calculation, however Penzien method 
leads to underestimation axial force in no-slip condition.

4) The comparisons clearly demonstrate that the Wang's solution provides a realistic estimate of the thrust in the tunnel linings for the no-slip condition. It is recommended that the Penziens's solution not be used for no-slip condition.

5) The modeling done by finite element method with the results of Wang, Penzien and bobet were compared. Despite the difference between the axial force of tunnel lining, the bending moment is close to the results of Wang.

6) About the axial force, the highest values obtained from numerical models. Results of the analytical methods, except for the results of Penzien is closer to each other. However, difference between the results of numerical modeling is significant.

7) The results of numerical modeling predict the axial force and moment in the tunnel lining, due to ovaling deformation under seismic stimulation, to assess the structural stability of the lining during earthquake. That the maximum bending moment with the results of Wang and Penzien have a great match, but there is more difference in the amount of axial force with the results of Wang and Bobet.

\section{References:}

A, Amorosi. D, Boldini. G, Falcone. “Numerical prediction of tunnel performance during centrifuge dynamic tests" Acta Geotechnica, 25 November 2013, Springer, Verlag Berlin Heidelberg, DOI 10.1007/s11440-013-0295-7, 2014.

Brady BHG, Brown ET. Rock mechanics for underground mining. London: Allen \& Unwin, 1985.

Carranza-Tores C, Fairhurst C. The elastoplastic response of underground excavations in rock masses that satisfy the Hoek- Brown failure criterion. Int J Rock Mech Min Geomech Abstr 1999;36:777-809.

Changguang Zhang, Junhai Zhao, Qinghe Zhang, Xiangdong Hub, A new closed-form solution for circular openings modeled by the Unified Strength Theory and radiusdependent Young's modulus, Computers and Geotechnics 42 (2012) 118-128.

D, De-yun. L, Wei-ning. S, GUPTA. G, LOMBAERT. G, DEGRANDE. "Prediction of vibrations from underground trains on Beijing metro line 15" J. Cent. South Univ. Technol. 17, pp. 1109-1118.2010.

D, Wu. B, Gao. "Dynamic Analysis of Tunnel Entrance Under the Effect of Rayleigh Wave" EJGE, Vol. 18, Bund. S, pp. 4231-4246. 2013.

Gercek H. An elastic solution for stresses around tunnels with conventional shapes. Int J Rock Mech Min Sci 1997;34(3-4), paper No. 096.

Gercek H. Stresses around tunnels with arched roof. Proceedings of the seventh International Congress on Rock Mechanics, vol. 2. Balkema, Rotterdam, The Netherlands: ISRM, 1991. p. 1297-99.

Hashash Y. M. A., Hook J. J., Schmidt B. \& Yao J. I., (2001). Seismic design and analysis of underground structure. Journal of Tunneling and Underground Space Technology 16, 247293.

Hashash Y. M. A., Park D. \& Yao J. I., (2005). Ovaling deformations of circular tunnels under seismic loading: an update on seismic design and analysis of underground structures. Journal of Tunneling and Underground Space Technology 20, 435-441.

Hoek E, Brown ET. Underground excavations in rock. London: Institute of Mining and Metallurgy, 1980.

Jaeger JC, Cook NGW. Fundamentals of rock mechanics, 2nd ed. London: Chapman \& Hall, 1976.

Kirsch G. Die theorie der Elastizit.at und die bed. urfnisse der festigkeitslehre. Zeit Ver Deut Ing J 1898;42:797-807.

Muskhelishvili NI. Some basic problems of the mathematical theory of elasticity. Groningen, The Netherlands: Noordhoof Ltd, 1963. 
Novozhilov VV. Theory of elasticity. New York: Pergamon Press, 1961.

QI, Taiyue. B, GAO. "Strata consolidation subsidence induced by metro tunneling in saturated soft clay strata" Journal of Modern Transportation Volume 19, Number 1, March, pp. 35-41. 2011.

S,A, Mazek. H,A, Almannaei. "Finite element model of Cairo metro tunnel-Line 3 Performance" Ain Shams Engineering Journal 4, pp.709-716.2013.
Sulem J, Panet M, Guenot A. An analytical solution for timedependent displacements in a circular tunnel. Int J Rock Mech Min Geomech Abstr 1987;24(3):155-64.

The Manh Vu, Jean Sulem, Didier Subrin, Nathalie Monin," Semi-Analytical Solution for Stresses and Displacements in a Tunnel Excavated in Transversely Isotropic Formation with Non-Linear Behavior" Rock Mech Rock Eng (2013) 46:213-229. 